\title{
Worst-Case Scenarios and Epidemics
}

\author{
G. Chowell and C. Castillo-Chavez \\ Center for Nonlinear Studies \\ Los Alamos National Laboratory \\ Los Alamos, NM 87545 \\ $\&$ \\ Biological Statistics and Computational Biology \\ Cornell University \\ Ithaca, NY 14853
}

February 17, 2003

\begin{abstract}
Contingency plans and policies associated with the deliberate release of biological agents must be developed in the absence of data. Hence, the identification of landscapes that facilitate "worst" epidemics (worst-case scenarios) is essential. Common sense suggest that "worst" epidemics are most likely to occur in populations where individuals mix randomly (proportionate mixing). Here, SIR (susceptible-infective-recovered) epidemics that result from the introduction of single or multiple sources are studied on various topologies including small-world and scale-free networks. A simple algorithm is used to compute the average growth rate during the initial exponential growth phase of the epidemic and such a rate is used to estimate the severity of the outbreak. In small-world networks, this average rate of epidemic growth is measured on the full spectrum of the disorder parameter $p \in[0,1]$. Extensive simulations show that the average rate of growth increases in a nonlinear fashion as the disorder in the network grows. Not surprisingly, simulations show that such average rate of growth is higher when the initial infective source is placed in the most connected node (pressure point) than when it is randomly placed. The average rate of initial growth is a non-decreasing function of the (small) number of initial infectious sources. Simulation results support the view that, worst-case
\end{abstract}


epidemics, in small-world networks, occur when the outbreak begins at a few places and when $p=1$ (random mixing). Simulation results also show that worst-case epidemics, on scale-free networks, are primary driven by the network hierarchy, that is, sources placed on the most connected nodes have the biggest effect. Finally, simulations suggest that a significantly higher average rate of epidemic growth is observed in scale-free than in smallworld networks. Hence, scale-free topologies may in fact provide the "best" landscapes for the theoretical study of worst-case epidemics.

\section{Introduction}

The potential deliberate release of biological agents such as small-pox,influenza, or foot and mouth disease (FMD) is a source of continuous concern to those responsible for the protection of our society. Contingency plans and policies must be developed in the absence of data. Hence, the identification of landscapes that facilitate "worst" epidemics (worst-case scenarios) is essential. It is believed that "worst" epidemics are most likely to occur in populations where individuals mix randomly (proportionate mixing). Therefore, the definition and indentification of landscapes or topologies that support worst-case scenarios is critical. Here, SIR (susceptibleinfective-recovered) epidemics that result from the introduction of single or multiple infectious sources are studied on various topologies including small-world and scale-free networks.

Most of the models studied in classical mathematical epidemiology fall in the class of compartmental models. The population under consideration is divided into classes or compartments determined by their epidemiological status [2]. The simplest version assumes that individuals mix uniformly (homogeneous mixing) within each compartment. The rates of transfer between compartments are expressed as derivatives with respect to time of the sizes of the compartments each mathematically represented as a differential equation. The study of the transmission dynamics of communicable diseases in human populations via mathematical (compartmental) models can be traced back to the work of Kermack and Mackendrick (1927). Their simple SIR (Susceptible-Infective-Removed) epidemic model (see Figure 1) was not only capable of generating realistic single-epidemic outbreaks but also provided important theoretical insights.

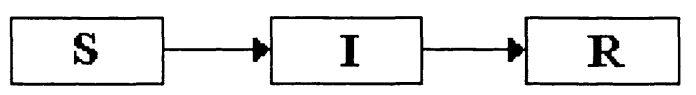

Figure 1: Simple SIR epidemic model. 
The Kermack and Mackendrick (K-M) model in essence captures the theoretical underpinnings associated with the framework that it is currently used to define and model worst-case epidemics outbreaks. The K-M model is given by the following system of nonlinear differential equations

$$
\begin{aligned}
& \frac{d S}{d t}=-\lambda(N) S I, \\
& \frac{d I}{d t}=\lambda(N) S I, \\
& \frac{d R}{d t}=\gamma I,
\end{aligned}
$$

where $S(t)$ denotes susceptible individuals at time $t ; I(t)$ infected (assumed infectious) individuals at time $t ; R(t)$ recovered (assumed permanently immune) individuals at time $t ; \lambda(N)$ the transmission rate when the total population is $N(N=S+I+R)$; and $\gamma$ denotes the recovery rate. In the case of a fatal disease, $N=S+I ; R(t)$ would denote those removed by death; and $\gamma$ would denote the per-capita death rate. Single outbreaks of communicable diseases such as smallpox, influenza, measles and rubella have been modeled using this framework.

The Kermack and Mackendrick threshold theorem established quantitatively the conditions required for successful disease invasion. Under the K-M model, this threshold theorem says that a disease would invade provided that its basic reproductive number

$$
R_{o}=\frac{\lambda(N(0)) N(0)}{\gamma}>1
$$

$R_{o}$ is interpreted as the number of secondary infectious individuals generated by a "typical" infectious individual when introduced into a fully susceptible population ([3],[4]). An alternative interpretation is that, in a randomly mixing population, a disease would invade provided that there are enough susceptibles, that is, if

$$
N(0)>\frac{\gamma}{\lambda(N(0))}
$$

Typically, it has been assumed that either $\lambda(N)=\frac{\beta_{o}}{N}$ or $\lambda(N)=\beta_{o}$ and, consequently, the "exact" interpretation of $R_{o}$ depends on the definition of $\lambda(N)([1],[2],[3])$.

Nold [5] introduced the concept of proportionate mixing as a way of introducing a simple form of heterogenous mixing in models for disease spread. She divided the population into $K$ 


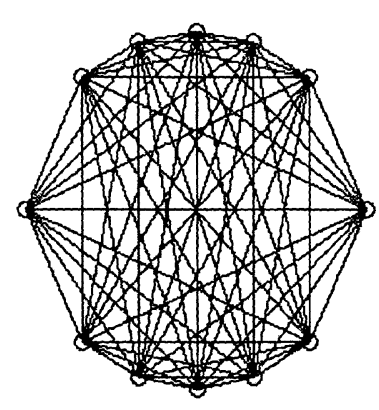

Figure 2: Fully-mixed transmission network.

groups each with population size $N_{i}(t)=S_{i}(t)+I_{i}(t)+R_{i}(t), \quad i=1, \ldots, k$.

Furthermore, if $P_{i j}(t)$ denotes the proportion of individuals of group $i$ who have a contact with individuals of group $j$ given that they had a contact with a member of the population at time $t$ then Nold's proportionate mixing corresponds to the case

$$
P_{i j} \equiv \bar{P}_{j}=\frac{C_{j} N_{j}}{\sum_{l=1}^{k} C_{l} N_{l}}
$$

where $C_{l}$ denotes the average activity level (contact rate) of individuals in group $l=1, \ldots, k$. Other forms of mixing can be found in [3], [6], [7], [8] and references therein.

One of Nold's version of the Kermack-Mackendrick model assumes that $\lambda_{j} \equiv \frac{\beta_{j}}{N_{j}}, \quad j=$ $1, \ldots, k$. The full system of nonlinear ordinary differential equations is

$$
\begin{aligned}
\frac{d S_{i}}{d t} & =-S_{i}(t) \sum_{j=1}^{k} \beta_{j} \bar{P}_{j} \frac{I_{j}}{N_{j}}, \\
\frac{d I_{i}}{d t} & =S_{i}(t) \sum_{j=1}^{k} \beta_{j} \bar{P}_{j} \frac{I_{j}}{N_{j}}-\gamma_{i} I_{i}, \\
\frac{d R_{i}}{d t} & =\gamma_{i} I_{i} .
\end{aligned}
$$

Examples of the analyses of compartmental models of this type can be found in [1],[2],[3], and [5]. Other types of compartmental models have considered local populations interconnected via migrating individuals. These models fall within the class of what is known as metapopulation models (see [8] and references therein). In the K-M model and Nold's models, individuals mix 
at random (see Figure 2), an uncommon situation. It has often been assumed that randomly mixing models support worst-epidemic outbreaks and recent analysis of worst-case scenarios, for the deliberate release of biological agents (smallpox in particular), has been carried out under such an assumption [13]. The focus of this paper (as suggested to us by Ed Kaplan) consists on the examination of this assumption.

The study of the meaning of worst-case scenarios using "mean" field models like the K-M or Nold's model is problematic. The modeling (mixing) assumes a predetermined number of groups (types) and worst-case scenarios results require an approach that consider all measures of mixing ([5]). Instead, we look at this question in the context of individual-based models where mixing is naturally embedded in the chosen topology. The goal of this work is to explore the impact of various topologies on the average rate of epidemic growth.

\section{Individual-based models}

Individual-based models must bring an explicit population structure on which disease dynamics are superimposed. Population structures are represented through networks (graphs) composed of nodes (individuals) and edges (representing predefined relationship between nodes). Examples include family trees which provide a simple picture of the direct descendants of a pair of individuals; traffic networks that describe street intersections by nodes, traffic direction by arrows (edges); and airport networks where nodes represent airports and edges connections between nodes. Graphs (networks) can be represented by a matrix or a list, the adjacency matrix or list. The adjacency matrix is an nxn matrix $T$ where $T(i, j)=1$ indicates that vertex $i$ is connected to vertex $j$. If the network is undirected (edges have no direction) then $\mathrm{T}$ is symmetric, that is, $T=T^{t}$ (transpose of $T$ ). An adjacency list representation simply lists the vertices of a graph and, next to each, the vertices adjacent to such a vertex [15]. The analysis of network models can be traced back to the work of Erdös and Rényi in the 1960's. These researchers introduced a simple algorithm for the construction of random networks [15]. We start with a fixed number of disconnected nodes $N$. Each pair of nodes is connected independently with an edge with probability $p_{E R}$ (not connected with probability $1-p_{E R}$ ). Hence, $p=0$ corresponds to the case where no node is connected to any of the other $N-1$ nodes. While $p_{E R}=1$ corresponds to the case where every node is connected to all other nodes in the network (complete graph). The total number of edges when $p_{E R}=1$ is $\left(\begin{array}{c}N \\ 2\end{array}\right)$. In general, the average number of edges in a network will be given by $\frac{N(N-1) p_{E R}}{2}$ while the average degree (number of edges incident from a node) of a node will be $z=(N-1) p_{E R} \simeq N p_{E R}$ (for large 
$N)$.

Erdős and Rény [15] showed that for large systems (large N) the probability that a node has $k$ ' edges follows the Poisson distribution $P(k)=\frac{\exp (-z) z^{k}}{k !}, \quad k=0,1, \ldots, N$.

Mathematically and computationally attractive is the phase transition observed with increasing $z$ (Poisson rate). There is a critical value of $z\left(z_{c}\right)$ such that whenever $z>z_{c}$, a connected component (a subset of vertices in the graph each of which is reachable from the others by some path through the network) forms. Such component is referred as the spanning cluster [36].

The Erdős and Rényi random graph model provides a null-model for the study of network topologies. The case $p_{E R}=1$ (totally connected network) is naturally seen as the generator of the landscape most conducive to disease spread. Such an assumption has been strengthened by the fact that random networks fail to describe real world networks. Highly-clustered networks have a higher probability that the neighbors of a particular node are also neighbors of each other. Hence, small-world networks have a short average distance between nodes, that is, the average number of edges needed to traverse in order to reach a node from any other node in the network is small. Watts and Strogatz (1998) [18] used data on real networks that show high degree of aggregation (clustering), a characteristic absent in random networks (see Erdős and Rényi,[15])
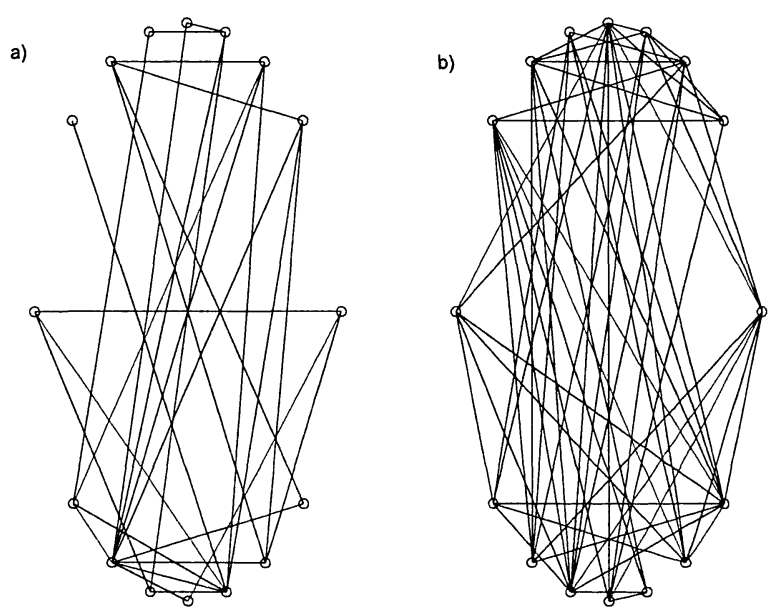

Figure 3: The Erdős and Rényi random graph with $N=16$. a) $p_{E R}=0.25$ b) $p_{E R}=0.5$

Watts and Strogatz (1998) [18] classified networks by their level of randomness, as measured by their own disorder parameter $p_{W S}$ (from "regular" $p_{W S}=0$ to completely random, $p_{W S}=1$ ). Whenever each node in a network is connected to its nearest two neighbors on its right and 

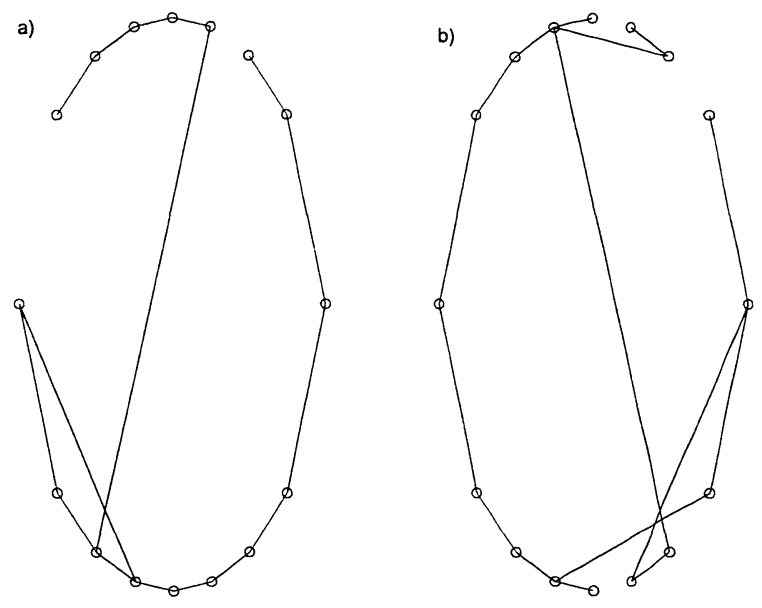

Figure 4: Small-world networks with $N=16, K=1$, a) $p=0.1$ b) $p=0.3$

to its nearest two neighbors on its left then the resulting network will be regular [18]. On the other hand, a completely random network has $p_{w S}=1$, all nodes are randomly connected to each other as in the Erdős and Rényi model.

Watts and Strogatz (1998) [18] took a one-dimensional ring lattice of $N$ nodes connected to its $2 K$ nearest neighbors ( $K$ is known as the coordination number) and periodic boundary conditions. Their algorithm goes through each of the edges in turn and independently with some probability $p_{W S}$ "rewires" it to a randomly selected node. That is, shifts one end of the edge to a new node chosen at random from the whole lattice (except that no two nodes can have more than one edge running between them, and no node can be connected by an edge to itself (see Figure 12).

Watts and Strogatz showed that the introduction of a small number of random connections $(p \simeq 0.01)$ in their regular networks significantly reduces the average distance between any two nodes (characteristic path length). In fact, they showed that such average distance grows like $O(\log (N))$ instead of $O(N)$. Furthermore, high levels of clustering were achieved in smallworld networks. The small-world effect (short average distance between nodes and high levels of clustering) has been detected in several networks including a network of actors in Hollywood, the power generator network in the western US, and the neural network of C.elegans. This small-world effect had been documented by the psychologist Stanley Milgram using the data from the letter-passing experiments that he conducted in the 1960s [16].

Newman and Watts [29] studied a slight variation of the Watts-Strogatz model. They added shortcut edges with probability $\phi$ per edge in the underlying ring latice instead of 'rewring' 


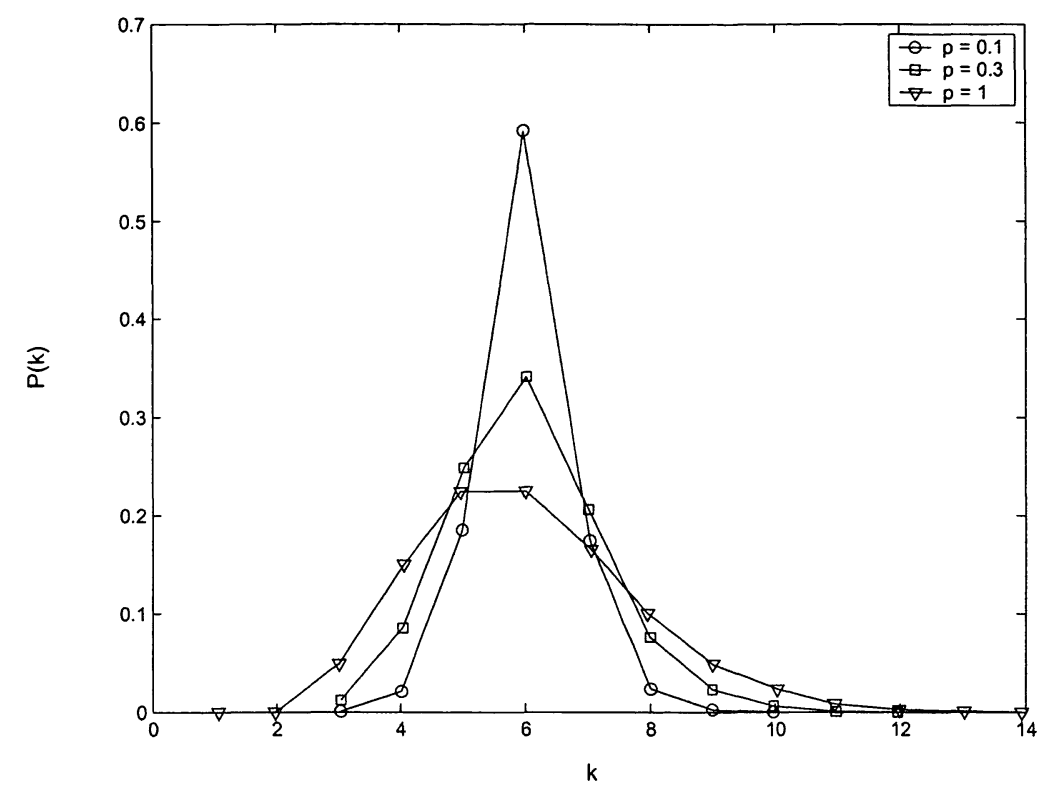

Figure 5: Connectivity distributions $P(k)$ of the small-world network model with three different disorder parameters $p=0.1, p=0.3$, and $p=1$ with networks of size $10^{4}$ and $K=3$.

the existing edges. The Newman and Watts model is easier to analyze since the network does not become disconnected after rewiring. Figure 6 shows a small-world network with $N=16$, $K=1$ and $\phi=0.1$. The degree or connectivity distribution of the small-world network model depends on the disorder parameter $p$. If $p=0$, the connectivity distribution is given by the delta function $\delta(k-2 K)$ where $K$ is the coordination number in the network. As $p$ approaches 1 , the connectivity distribution converges to that obtained from the Erdoss and Rényi model. Figure 5 shows the degree distribution for the small-world model at various values of the disorder parameter $p$ for networks of size $10^{4}$ and $K=3$. The bell-shaped node degree distributions observed in the Erdős-Rényi, the Watts-Strogtaz, and the Newman-Watts models are in contrast with the power-law degree distributions observed in a number of biological [48], social [19] [20], [23] [30] [31] [43] [47], and technological [19] [20] [49][50] networks (see Figure 7). Power-law degree distributions (also known as the Pareto distribution among statisticians) are given by the parametric family:

$$
P(k)=C k^{-\gamma}
$$

where $\gamma$ is typically between 2 and 3 (infinite variance) and $C$ is a normalization constant (makes $P(k)$ a probability density function). Networks that fit well power-law degree distributions have 

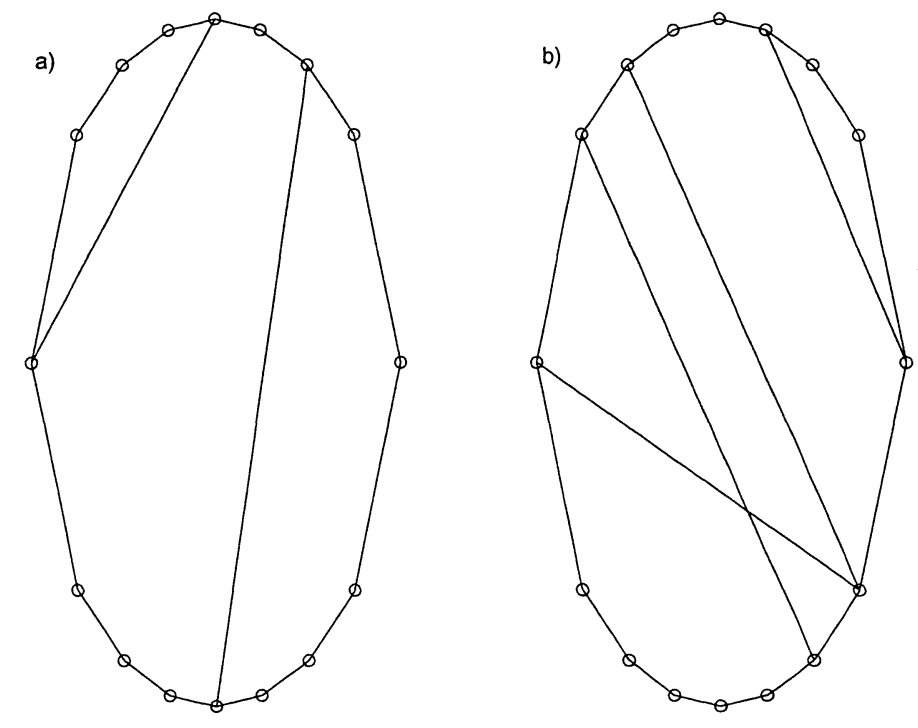

Figure 6: Small-world networks with $N=16, K=1$, a) $\phi=0.1$ b) $\phi=0.3$

a small number of highly connected nodes, that is, most of the nodes have a small number of connections. Barabási and Albert (1999) have dubbed this type of structure scale-free networks. Scale-free network structures have been observed in various sets of data. The number of sexual partners in the 1996 Swedish survey of sexual behavior [43] fits a power-law distribution. the spread of sexually-transmitted diseases such as HIV. The data from the number of sexual partners of Cornell University undergraduates from the 1990 Cornell Undergraduate Social and Sexual Patterns (CUSSP) survey [46] can also be fitted well by such distributions. Those results strengthen the view that sex-education campaigns should target individuals with the highest number of partners (core group) [10]. The location-based network of the city of Portland, Oregon also exhibits a scale-free structure (Chowell et al. [47]). Here, nodes represent locations while directed connections between locations represent the average movement of individuals in the city. The scale-free (Figure $7(\mathrm{~d})$ ) topology implies the existence of a high a number of locations with a low number of connections (i.e households) and a small number of highly connected hubs (i.e schools, hospitals, etc.). Barabási and Albert (1999) introduced a simple theoretical model that generates networks with a power-law degree distribution (see Figure 10) [19][20]. The BA algorithm starts with a small number of nodes $\left(m_{o}\right)$. At each time step nodes connect (with $m$ links), with higher probability, to nodes that have accumulated a higher number of connections. The resulting network has a power-law exponent of 3 and a mean connectivity of $2 m$. Thus the Barabási-Albert (BA) model captures features that are 

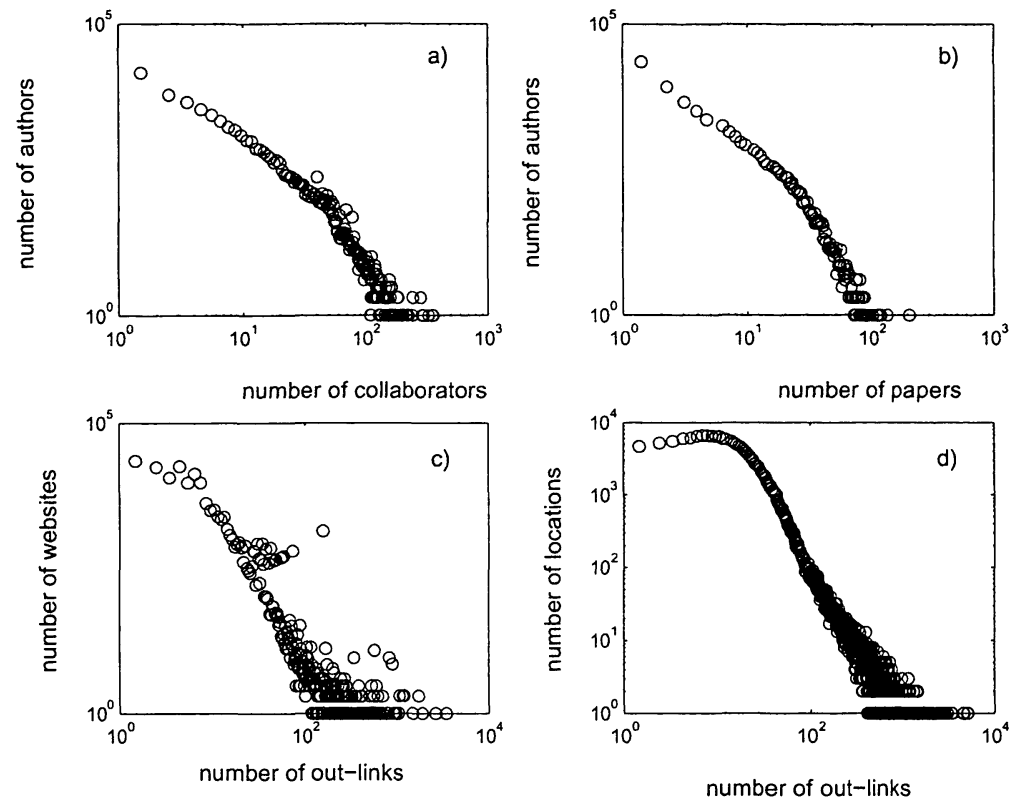

Figure 7: The power-law distributions observed in a,b) Scientific collaboration networks (The Los Alamos e-Print Archive) [51] c) The World-Wide-Web (nd.edu domain) [52] and d) The location-based network of the city of Portland [47]

characteristic of real-world networks: growth and prefferential attachment.

Figure 9 shows a network generated using the BA model. Several modifications of the BA model have been studied including edge rewiring [24], edge removal [25], growth constraints [28][26], and edge competition [27]. Klemrn and Eguiluz (2002) [21] developed an altervative model that also generates scale-free networks. These researchers incorporated memory as part of a node's ability to acquire more links. The Klemrn-Eguiluz model produces scale-free networks with a high clustering coefficient, a property not generated by the BA model.

The capacity of networks to mantain essential properties when some nodes are removed is a measure of network robustness. In scale-free networks, most of the nodes have low degree, hence their removal does not impact the connectivity of the remaining vertices. However, the removal of nodes with the highest degree (pressure points) of connectivity can have dramatic consequences. In fact, it can totally alter the network's connectivity structureThe removal of highly connected can totally alter the network's connectivity structure. This effect was first demonstrated independently and numerically by Albert (2000) and Broder (2000) using subsets of data of the World-Wide Web. The practical relevance of network robustness was highlighted 

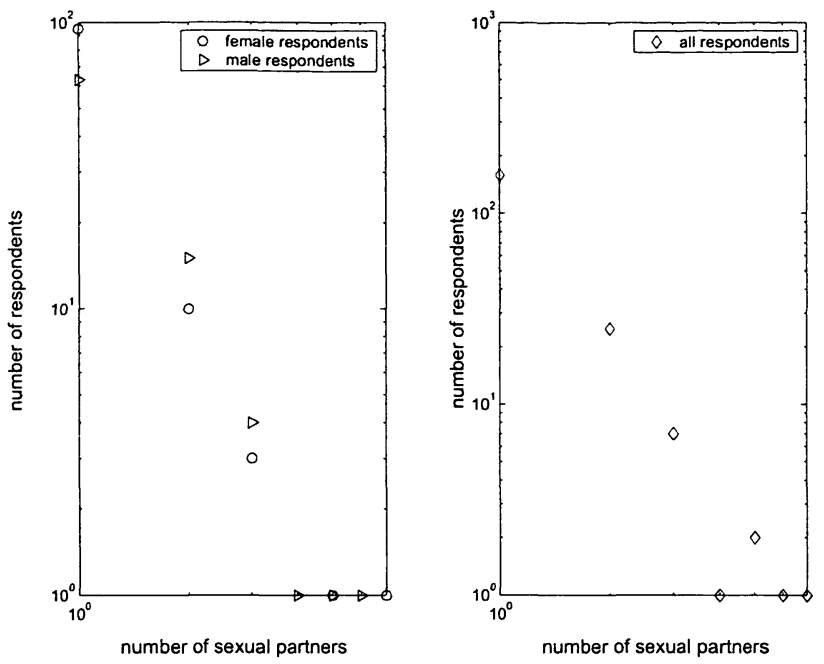

Figure 8: Number of sexual partners of Cornell University undergraduates from the 1990 Cornell Undergraduate Social and Sexual Patterns (CUSSP) survey[46]. The power-law exponent for females is 2.86 , for males is 2.90 , and for the overall distribution is 2.78

by the recent service denial of highly connected web servers including Yahoo, CNN, Amazon, Ebay, Excite and Etrade following a network attack.

Important work on the use of worst-case scenarios in the development of response policy has been carried out by Kaplan [11],[12],[13] in the context of HIV and smallpox. However, the nature of his approach does not allow for the incorporation of population structures such as those identified in [19] [20], [23], [30], [31], [47]. The focus of this paper (instigated by Ed Kaplan) is driven by these questions:

How is the initial rate of growth of epidemics affected by a population's structure? What is the role of 'social' topologies and the number of initial infectious sources on the rate of growth of an epidemic?

The following sections represent our initial attempts to address these questions in the context of small-world [17] [18] and scale-free networks [19]. The organization of the rest of this paper is as follows. Section II corroborates Kaplan's view of mixing in worst-case epidemics when the transmission topology is given by small-world networks. In Section III, the study of epidemics in scale-free networks where a natural "node" hierarchy often emerges, is studied. This structure, 


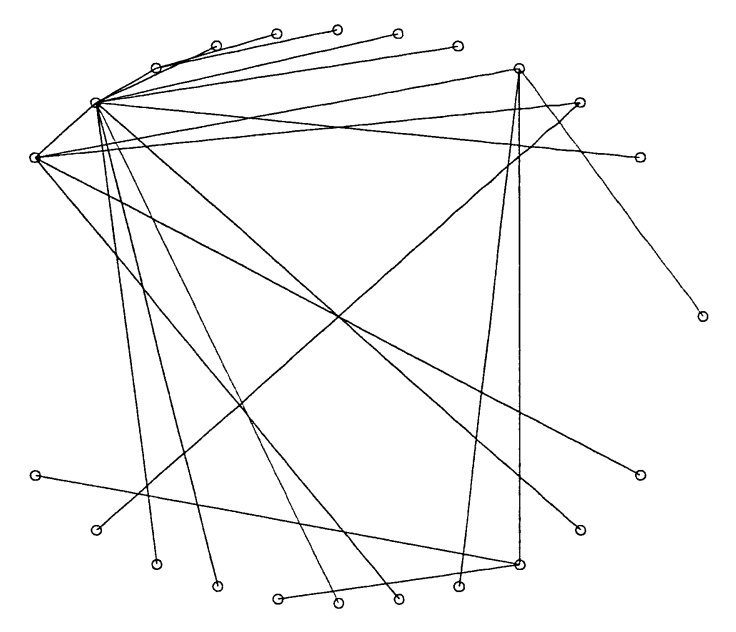

Figure 9: Network of size $N=23$ generated using the BA model described in the text with $m_{o}=2$ and $m=1$.

in some sense, "equivalent" to the concept of core group developed by Hethcote and Yorke (1984) [10], seems to provide a better landscape for disease spread. Section IV collects our conclusions and views on implications of these results in the study of the impact of deliberate releases of biological agents.

\section{$3 \quad$ Epidemics on small-world networks}

Simple epidemic models such as the susceptible-infected-recovered (SIR) model have been studied on small-world networks. Moore and Newman [34] studied SIR epidemics on small world networks via site and bond percolation. In site percolation, nodes (sites) are occupied (by spins) or not and any two spins occupying nearest neighbour sites are connected by an open bond. In bond percolation, the relevant entities are bonds or edges. Bonds are sequentially visited and set open with probability $p$ or closed with probability $1-p$ (independently). The percolation threshold is the smallest probability $p$ at which an infinite cluster of sites emerges when sites or bonds (depending on the type of percolation) are occupied with that probability.

SIR epidemic process are built on the assumption that nodes are occupied by individuals which can be infected by neighbors connected by edges or bonds (Grassberger [35], 1983). The epidemic threshold $R_{o}$ can be mapped to the corresponding percolation threshold $\left(p_{c}\right)$. When the total probability of transmission from one individual to another is greater than this 


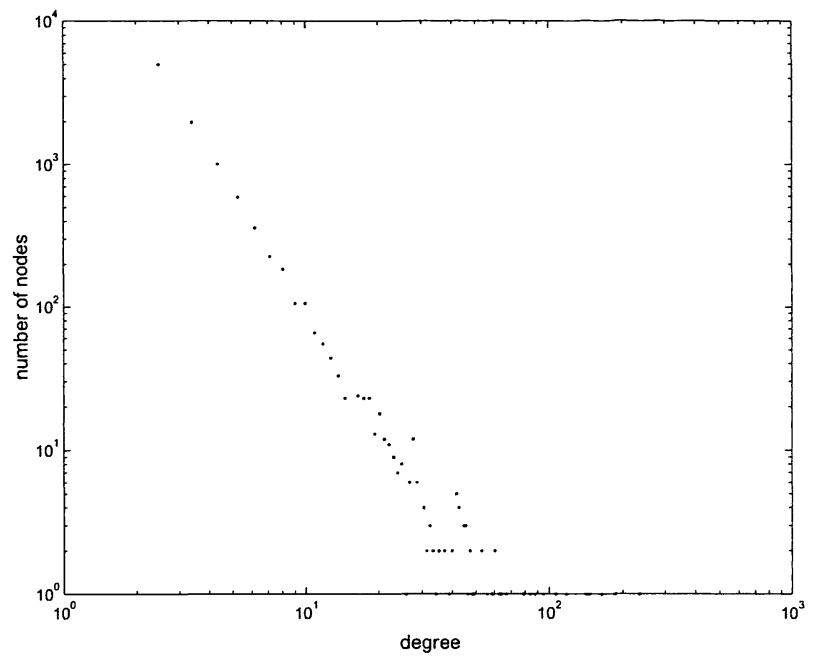

Figure 10: Connectivity distribution of the BA model decays as a power law with $\gamma=3$. Here, $N=10000, m_{o}=3$ and $m=2$.

threshold, the disease explodes, that is, a "giant" component (whose size is the size of the epidemic) appears. In an epidemic that starts with a single infectious source and spreads as a bond percolation process, the subset of nodes (individuals) that can be reached from the initial infective individual by traversing only open bonds, is the size of the epidemic outbreak. The study of plant epidemics requires the introduction of spatially explicit schemes as plants have a fixed position. Newman [33] studied SIR epidemics on a two-dimensional small world network via bond percolation. His results were motivated by the study of disease transmission in plants from nearest neighbor (plants) and long-distance contacts (vectors). Epidemics on small-world networks can exhibit phase transition behavior, that is, there is a critical value of the disorder parameter $\left(p_{c}\right)$ such that for values of $p>p_{c}$ self-sustained oscillations in the number of infected individuals in susceptible-infected-susceptible (SIS) epidemic models are possible (Kuperman et al. [37]).

In order to study the role of the disorder parameter $p$ (small-world networks) on the initial rate of growth of disease spread, the following algorithm is used to compute its initial (empirical) rate of growth of an SIR epidemic. The number of infected individuals is computed as a function of time $I(t)$ (time is discrete) for a small time range $\left(t<t_{c}\right)$. The value of $t_{c}$ is selected so that $I(t)$ is still in its exponentially growing phase. The algorithm follows three steps:

1. Computation of $t_{c}$. The time $t_{c}$ at which $I(t)$ changes cancavity, that is, the value of $t$ at which the second derivative of $\mathrm{I}(\mathrm{t})$ changes from positive to negative (see Figure 11). 


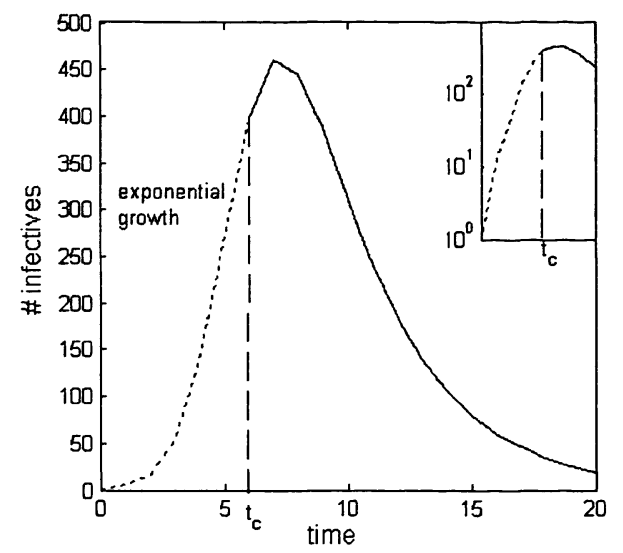

Figure 11: Computation of the empirical rate of growth of epidemics on networks.

2. Rescaling of $I(t)$. $\hat{I}(t) \equiv \log _{e}[I(t)]$ for $t<t_{c}$ where $t_{c}$ is the value computed in Step 1 .

3. Regression on $\hat{I}(t)$. Compute the average slope $r$ of the best fitting line to $\hat{I}(t) . \quad r$ is the average " $r$ " that results from 50 realizations. The average initial rate of growth $r$ is computed as a function of the disorder parameter $(p)$ of small-world networks $(p \in[0,1]$ is changed in increments of 0.01 ).

\subsection{Epidemiological model}

We consider an stochastic SIR epidemiological model. Hence, individuals can be in one of the three epidemiological states: Suceptible (S), infected (I), or recovered (R). A susceptible individual in contact with $i$ infectious individuals may become infected in a short period of time $\delta t$ with a probability given by,

$$
P_{S I}=1-e^{-\hat{\beta} I_{n} \delta t}
$$

where $\hat{\beta}$ is the constant risk of infection per unit of time and $\delta t=1$ in this discrete time model. Similarly, infected recover with a probability given by 


$$
P_{I R}=1-e^{-\hat{\gamma} \delta t}
$$

where $\frac{1}{\hat{\gamma}}$ is the mean period of infectivity. After recovery, individuals get full immunity to the disease.

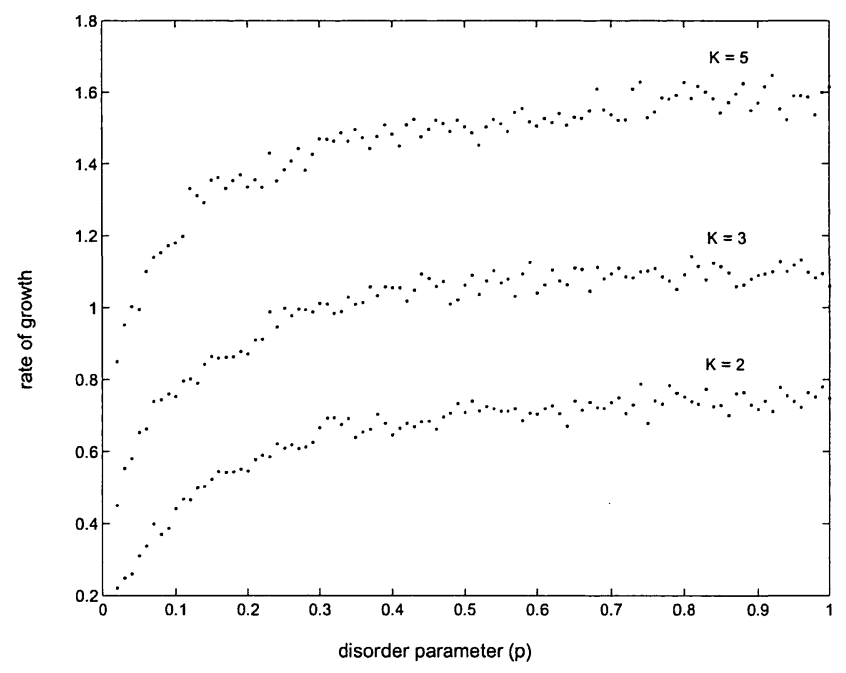

Figure 12: Rate of growth of epidemics in small-world networks of size $N=10^{3}$ with $K=2$, $K=3$, or $K=5$, and as a function of the disorder parameter $p \in[0,1]$. Averages are taken from 50 realizations. $p$ is incremented by 0.01 . Disease parameters are: $\hat{\beta}=\frac{4}{7}, \gamma=\frac{2}{7}$ and $I(0)=1$

Epidemics were simulated on small-world networks of size $N=10^{3}$ with $K=2, K=3$, and $K=5$ ( $\mathrm{K}$ is the coordination number of small-world networks). Empirical results on the average rate of growth were obtained from the mean of 50 realizations with disease parameters $\hat{\beta}=\frac{4}{7}$ and $\hat{\gamma}=\frac{2}{7}$. Simulations were started by placing randomly a single infectious source. The average rate of growth increases in a nonlinear fashion as the disorder in the network grows. It saturates when it is close to 1 (totally random networks) with $r_{\text {random }} \approx 0.7481$ and $K=2$. Figure 12 shows the average (from 50 realizations) rate of growth of epidemics in small-world networks of size $N=10^{3}$ (with $K=2, K=3$, and $K=5$ ) as a function of the disorder parameter $p$. The rate of growth in small-world networks also increases as the coordination number $(K)$ increases. 


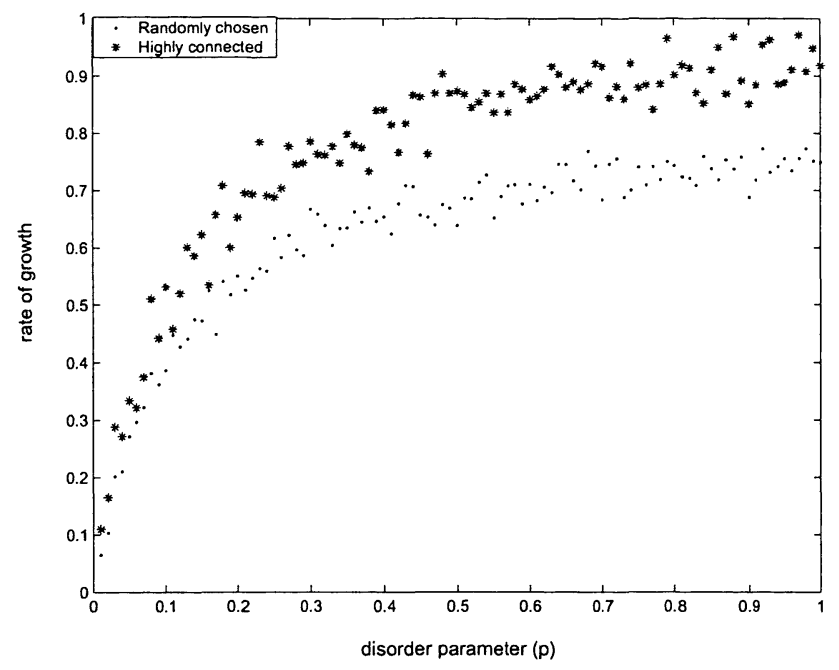

Figure 13: Rate of growth is higher when the epidemics start at the individuals (nodes) with the highest connectivity $\left(^{*}\right)$ rather than chosen uniformly at random (.) with $N=10^{3}$ and $K=2$. Averages are taken from 50 realizations. Disease parameters are: $\hat{\beta}=\frac{4}{7}, \gamma=\frac{2}{7}$ and $I(0)=1$.

The initial rate of growth of epidemics depends on the network topology. The following simulations (to highlight this observation) start with and initial (small) group of infective individuals chosen from those with the highest connectivity. The resulting rate of growth is computed and compared to that resulting from epidemics where the intial infectious sources are chosen (uniformly) at random. Naturally, epidemics that started at the most connected nodes exhibited a higher average rate of growth (see Figure 13). Higher rates of growth are observed as the number of initial infectious sources (always small compared to the size of the network) in the network increases (see Figure 14).

\section{Epidemics on scale-free networks}

Pastor-Satorras and Vespignani [39] studied a simple SIS epidemic model on scale-free networks (generated using the BA model) and found that a disease may persist independently of its transmissibility. That is, the basic reproductive number $R_{o}$, routinely computed in classical mathematical epidemilogy, sometimes loses its meaning in this setting. The small number of nodes with a high connectivity ( $h u b s$ ) observed in scale-free networks are responsible for "zero" threshold behavior. This observation gives rise to the following question: Can a control strategy 


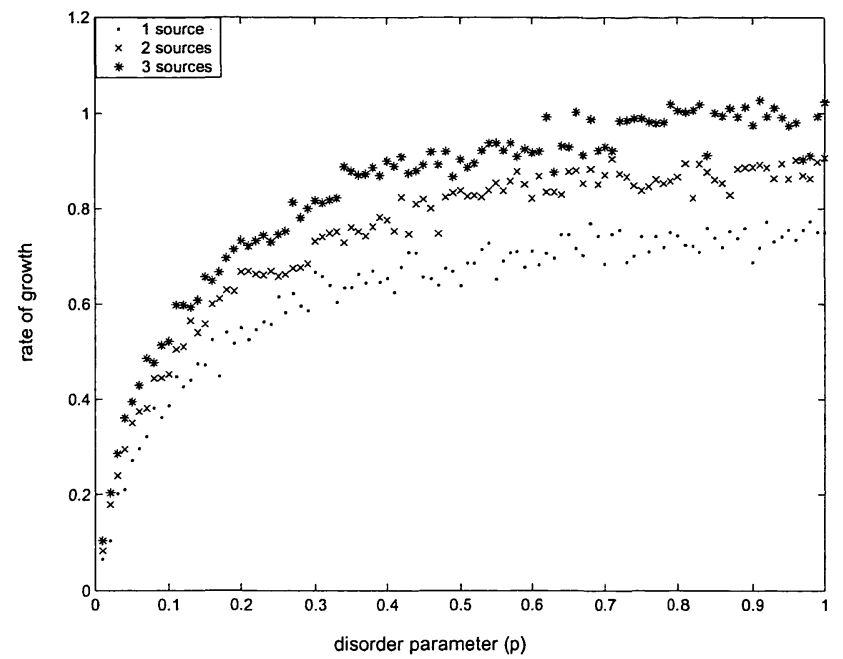

Figure 14: Average rate of growth of multiple source epidemics: one source $[I(0)=1]($.$) , two$ sources $[I(0)=2](\mathrm{x})$ and three sources $[I(0)=3]\left({ }^{*}\right)$ with $N=10^{3}$ and $K=2$. Averages are taken from 50 realizations. Disease parameters are: $\hat{\beta}=\frac{4}{7}, \gamma=\frac{2}{7}$.

be implemented that restores a positive epidemic threshold?

This question was studied independently by Pastor-Satorras and Vespignani [40] and by Dezso and Barabási [42]. Both groups concluded that targeted immunization campaigns towards the most connected nodes or hubs increase the probability of recovering finite epidemic threshold behavior. A contrasting result has been established on altervative highly clustered scale-free networks [21]. Here, a finite epidemic threshold has been observed on (SIS) epidemics (Eguíluz and Klemrn [22]).

An extensive number of simulations has been carried out using an epidemiological SIR model on scale-free networks constructed via the BA model. We compute the average rate of growth from the mean of 50 realizations of the epidemic process with two sets of initial conditions: We place the infective source at a randomly selected node or at the most connected node (highest degree). Simulations are carried out on small-world and scale-free networks of the same size $\left(N=10^{3}\right)$ and average connectivity $(\bar{k}=4)$. Significantly higher rates of growth are observed in scale-free networks (see Figure 15). Hence, scale-free topologies seem to provide ideal scenarios for the study of worst-case epidemics. The existence of highly connected nodes or hubs in scalefree networks plays a central role on the rate at which viruses (or information) spreads. Or, in other words, the concept of core group [10], is still critical to disease spread on topologically defined networks. 


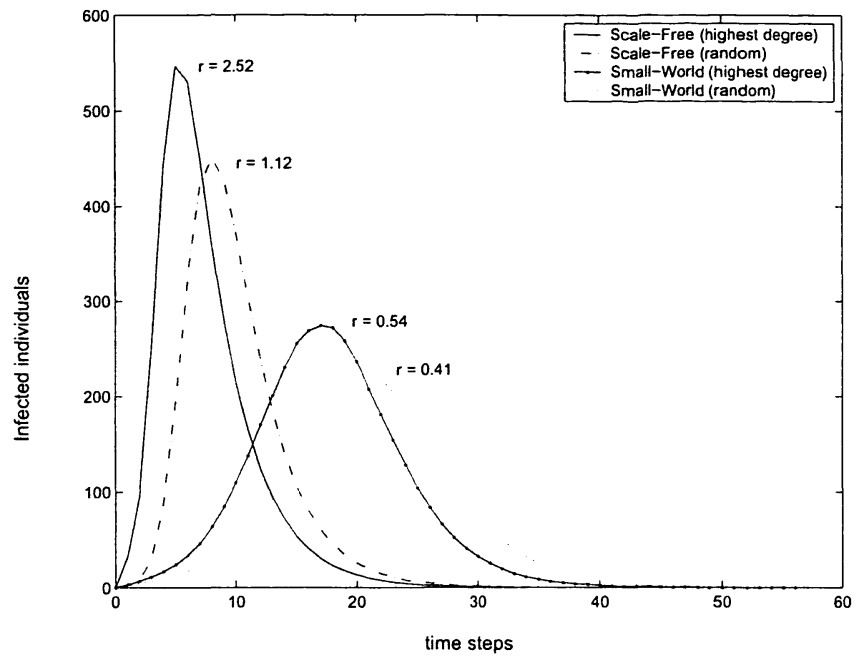

Figure 15: Average number of infected individuals from 50 realizations over time in small-world and scale-free networks of the same size $\left(N=10^{3}\right)$ and average connectivity $(\bar{k}=4)$. Two different initial conditions are considered: The initial infectious source is placed in a randomly selected or in the most connected node (highest degree). The rates of growth of the epidemics are higher in scale-free networks. Disease parameters are: $\hat{\beta}=\frac{4}{7}, \gamma=\frac{2}{7}$ and $I(0)=1$.

\section{Conclusions}

The development and implementation of policies that deal with the deliberate release of biological agents must consider worst possible situations and such scenarios are highly dependent on the network of individual interactions (social topology). Hence, gaining some understanding of the nature of the topological social structures that facilitate disease spread is critical. Kaplan et al. [13] assumes that random mixing corresponds to a worst-case scenarios and (using such a set up) concludes, in the case of a smallpox bio-terrorist attack, that mass vaccination, is a better policy than ring vaccination. Halloran et al. [14] using a stochastic model with a structured community of 2000 people conclude that targeted vaccination outperforms mass vaccination. The disagreement in results may be directly related to the assumed population structure and mixing topology (network of interactions).

Here, we have tried to identify under what conditions random mixing can be used to support worst-case scenarios. We have found that on small-world networks, random mixing, indeed supports epidemics with the highest average rate of growth. However, this is not necessarily the case on scale-free networks. The nature of the mixing between individuals (the connectivity 
hierarchy in scale-free networks) also plays a key role on the initial average rate of growth of an epidemic. The inherent connectivity hierarchy of scale free networks and the sensitivity (lack of robustness) of such networks to the removal of key nodes (most connected individuals), in some sense, corresponds to the critical importance that the concept of core group [10] has played in classical epidemiology. Highly connected nodes are indeed pressure points in the network and, consequently, their identification and management must be considered in the development and implementation of a logistic plan of response to the threat of a bio-terrorist attack.

We are continuing to explore these ideas and hope to test them on simulated data. The location-based network of the simulated city of Portland possess scale-free nature (Chowell et al. [47]). That is, most locations in this simulated city have a small number of connections (to other locations) while a small number of locations (hubs) are very connected. Hence, the initial rate of growth of epidemics in this city is (on the average) significantly higher whenever a source is placed at a hub (see Figure 15).

While the use of classical epidemiological approaches has been and will continue to be quite useful [1][2]. It is clear that the study of of the potential impact of the deliberate release of pathogens on unsuspecting populations must be addressed on multiple set ups. The reasons for such an approach are multiple, the interest is no longer on the long-term disease dynamics and the need for policies that result on timely responses is critical. Such a degree of urgency requires the identification of the most sensitive points of release (pressure points) and the possibility of multiple releases. Unfortunately, when it comes down to situations where pathogens are released in a deliberate manner planning for the worst-case situation is not as farfetched as it may seem.

\section{References}

[1] R. M. Anderson and R. M. May, Infectious Diseases of Humans, Oxford University Press (1991).

[2] F. Brauer, C. Castillo-Chavez, Mathematical Models in Population Biology and Epidemiology, Springer Verlag New York (2000).

[3] O. Diekmann, J. Heesterbeek, Mathematical Epidemiology of Infectious Diseases: Model Building, Analysis and Interpretation, John Wiley \& Sons (2000). 
[4] Castillo-Chavez C., Z. Feng and W. Huang, On the computation Ro and its role on global stability, Mathematical Approaches for Emerging and Reemerging Infectious Diseases: An Introduction, IMA Volume 125, 229-250, Springer-Veralg, Berlin-Heidelberg-New York.

[5] A. Nold, Heterogenity in Disease Transmission, Math. Biosci. 52 227-240 (1980).

[6] S. Busenberg and C. Castillo-Chavez, Interaction, Pair Formation and Force of Interaction Terms in Sexually Transmitted Diseases, Mathematical and Statistical Approaches to AIDS Epidemics (1990).

[7] C. Castillo-Chavez, J.X. Velasco-Hernandez, and S. Fridman, Modeling Contact Structures in Biology, Frontiers of Theoretical Biology, Lecture Notes in Biomathematics, S. A. Levin (ed). 100 454-91 (1994).

[8] C. Castillo-Chavez and A. Yakubu, Intra-specific competition, dispersal and disease dynamics in discrete-time patchy environments, Mathematical Approaches for Emerging and Reemerging Infectious Diseases: An Introduction, IMA Volume 125, 165-181, SpringerVeralg, Berlin-Heidelberg-New York.

[9] C. Castillo-Chavez, A. Yakubu, H. Thieme, M. Martcheva, Nonlinear mating models for populations with discrete generations, Mathematical Approaches for Emerging and Reemerging Infectious Diseases: An Introduction, IMA Volume 125, 165-181, Springer-Veralg, BerlinHeidelberg-New York.

[10] H.W. Hethcote and J.A. Yorke, Gonorrhea Transmission Dynamics and Control, Lecture Notes in Biomathematics 56, Springer, Berlin, 1984, 105 pages, ISBN 0-387-13870-6.

[11] E. H. Kaplan, Asymptotic worst-case mixing in simple demographic models of HIV/AIDS, Math. Biosci. 108 141-56 (1992).

[12] E. H. Kaplan, Mean-max bounds for worst-case endemic mixing models, Math Biosci. 105 97-109 (1991).

[13] E. H. Kaplan, D. Craft, and L. Wein, "Emergency Response to a Smallpox Attack: The Case of Mass Vaccination", Proceedings of the National Academy of Sciences (2002).

[14] M. E. Halloran, I. M. Longini, Jr. A. Nizam, and Y. Yang, Containing Bioterrorist Smallpox, Science 298: 1428-1432 (2002).

[15] B. Bollobás, Random Graphs (1985) (Academic, London). 
[16] S. Milgram, The small world problem, Psychol. Today 2,60-67 (1967).

[17] S.H. Strogatz, Exploring Complex Networks, Nature 410, 268-276 (2001).

[18] D. J. Watts and S. H. Strogatz, Collective Dynamics of 'small-world' networks, Nature 383, 440-442 (1998).

[19] A.-L. Barabási and R. Albert, Emergence of Scaling in Random Networks, Science 286, 509-512 (1999).

[20] A.-L. Barabási, R. Albert, H. Jeong, Mean-field theory for scale-free random networks, PHYSICA A 272 173-87 (1999).

[21] K. Klemrn and V. M. Eguíluz, Highly clustered scale-free networks, Phys. Rev. E 65, 036123 (2002).

[22] V. M. Eguíluz and K. Klemrn, Epidemic threshold in structured scale-free networks, Phys. Rev. Lett. 89, 108701 (2002).

[23] A.-L. Barabsi, H. Jeong, R. Ravasz, Z. Nda, T. Vicsek, and A. Schubert, On the topology of the scientific collaboration networks, Physica A 311, 590-614 (2002).

[24] Albert R., and A.-L. Barabási, Topology of evolving networks: Local events and universality, Physical Review Letters 85, 5234 (2000).

[25] S.N. Dorogovtsev and J.F.F. Mendes, Language as an evolving Word Web, Proc. Royal Soc. London B 268, 2603-2606 (2001).

[26] S.N. Dorogovtsev and J.F.F. Mendes, Scaling properties of scale-free evolving networks: Continuous approach, Phys. Rev. E 63, 056125 1-19 (2001).

[27] G. Bianconi, and A.-L. Barabási, Competition and multiscaling in evolving networks, Europhys. Lett. 54436 (2001).

[28] Amaral, L. A. N., A. Scala, M. Bartélémy, and H. E. Stanley, "Classes of Small-World Networks", Proc. Natl. Acad. Sci. U.S.A 97, 21 (2000).

[29] M. E. J. Newman and D. J. Watts, Renormalization group analysis of the small-world network model, Phys. Lett. A 263, 341-346 (1999).

[30] M. E. J. Newman, The Structure of Scientific Collaboration Networks, Proc. Natl. Acad. Sci. 98, pp. 404-409 (2001). 
[31] M. E. J. Newman, Who is the best connected scientist? A study of scientific coauthorship networks, Phys Rev. E64 (2001) 016131; Phys.Rev. E64 (2001) 016132.

[32] M. E. J. Newman, The spread of epidemic disease on networks, Phys. Rev. E 66, 016128 (2002).

[33] M. E. J. Newman, Percolation and epidemics in a two-dimensional small world, Phys. Rev. Lett. 66, 021904 (2002).

[34] C. Moore and M. E. J. Newman, Epidemics and percolation in small-world networks, Phys. Rev. Lett. 61, 5678-5682 (2000).

[35] P. Grassberger, On the critical behavior of the general epidemic process and dynamical percolation, Math. Biosc. 63, 157-172 (1983).

[36] Dietrich Stauffer and Amnon Aharony, Introduction to Percolation Theory, Revised 2nd Edition, 2002.

[37] M. Kuperman and G. Abramson, Small-world effect in an Epidemiological model, Phys. Rev. Lett. 86, 2909-2912 (2001).

[38] C. P. Warren, L. M. Sander, and I. Sokolov, Firewalls, disorder, and percolation in epidemics, cond-mat/0106450.

[39] R. Pastor-Satorras and A. Vespignani, Epidemic dynamics and endemic states in complex networks, Phys. Rev. Lett. 63, 066117 (2001).

[40] R. Pastor-Satorras and A. Vespignani, Immunization of complex networks, Phys. Rev. Lett. 65, 036104 (2002).

[41] R. M. May and A. L. Lloyd, Infection dynamics on scale-free networks, Phys. Rev. Lett. 64, $066112(2001)$.

[42] Z. Dezso and A.-L. Barabási, Halting viruses in scale-free networks, Rev. Lett. 65, 055103 (2002).

[43] F. Liljeros, C.R. Edling, L. A. N. Amaral, H. E. Stanley, and Y. Aberg, The web of human sexual contacts, Nature (London) 411, 907 (2001).

[44] G. Chowell, C. Castillo-Chavez, and S.-F. HSU SCHMITZ, Power laws in the distribution of the number of sexual partners in the 1990 Cornell Undergraduate Social and Sexual Patterns (CUSSP) survey, manuscript (2002). 
[45] S. P. Blythe \& C. Castillo-Chavez, Nature 344202 (1990).

[46] C. M. Crwaford, S. J. Schwager, and C. Castillo-Chavez, Research design for the Cornell undergraduate social and sexual patterns survey, Technical report HSS-90-CC1/BU-1083-M, Human Service Studies, Cornell University, Ithaca, New York.

[47] G. Chowell, J. M. Hyman, S. Eubank, C. Castillo-Chavez, Analysis of a Real-World Network: The City of Portland, Los Alamos Unclassified Report LA-UR-02-6658, BSCB department Report BU-1604-M, Cornell University (2002).

[48] H. Jeong, B. Tombor, R. Albert, Z.N. Oltvai, and A.-L. Barabási, The large-scale organization of metabolic networks, Nature 407, 651-654 (2000).

[49] Ravi Kumar, Prabhakar Raghavan, Sridhar Rajagopalan, D. Sivakumar, Andrew S. Tomkins, Eli UpfalProc (200). 19th ACM SIGACT-SIGMOD-AIGART Symp. Principles of Database Systems, PODS.

[50] M. Faloutsos, P. Faloutsos, C. Faloutsos, On Power-Law Relationships of the Internet topology, SGCOMM (1999).

[51] http://www.nd.edu/ networks/database/index.html

[52] http://www.santafe.edu/ mark/collaboration/ 